\title{
3D PRINTING IN MEDICINE - PRINCIPLES, APPLICATIONS AND CHALLENGES
}

\author{
Petar Valchanov \\ Department of Anatomy and Cell Biology, Faculty of Medicine, \\ Medical University of Varna, Bulgaria
}

\begin{abstract}
INTRODUCTION: 3D printing is a fast developing, precise and reachable technology, which operates with a vast variety of materials and is used to build three-dimensional models with many purposes, including application in many fields of the medicine.

MATERIALS AND METHODS: 3D printing allows us to convert a tomographic image study or a schematic diagram into a digital 3D model. Then we can build layer by layer a solid replica of this model with a robot, called CNC machine. This machine can use virtually any available material, including metals and metal alloys, clays, natural or synthetic polymers, glass, textile, food or even living cells.

RESULTS: 3D printing can be used in medicine for the construction of highly accurate, anatomically correct models, both on microscopic and macroscopic level. Those models can be used as educational tools, as accurate training models or as custom, individualized medical tools and devices. Living cells can be included in the model, which results in a bioartificial tissue or organ model (organoid, bionic organ, bioimplant), which can be used for pathophysiological and pharmaceutical experiments or it can be implanted in a living host to replace a diseased or absent one.

CONCLUSION: 3D printing is a powerful tool, which potentially can recreate any structure of the body as an inanimate educational or training model or as a living organ, which can replace the functions of the natural one or even to augment them. This technology is still in its early stages of development, but it has an enormous potential and will change the medicine forever.
\end{abstract}

Keywords: 3D printing; 3D modeling, CNC, bioartificial organs

Address for correspondence:

Petar Valchanov

Faculty of Medicine

Medical University of Varna

55 Marin Drinov St

9002 Varna, Bulgaria

e-mail:petar.valchanov@mu-varna.bg

Received: November 2, 2017

Accepted: December 5, 2017

\section{INTRODUCTION}

Additive manufacturing is a building method, in which the desired material is added to a building platform, which results in the creation of a three-dimensional object. An early version of this method was developed in the antiquity by the ancient Romans, controlled by the hands of the crafters and the artisans, but in the 1980s this technology was scaled to a whole new level when a computer was added to 
provide precise control over the building process through a specially designed robot - the CNC machine. An entire industry grew around the technology, known as rapid prototyping, which was able to quickly fabricate a scale model of a physical object using a three-dimensional digital computer-aided design (CAD) model. This led to the so-called 3D printing revolution - an open source initiative, called the RepRap project - humanity's first general-purpose, self-replicating manufacturing machine. Professional engineers and self-taught amateurs alike started to improve the machine itself and its controlling software for decades, dramatically increasing its overall quality, the range of the building techniques, the available building materials and the reachability of the technology. While the technology was rapidly improved, the cost decreased substantially. Today it is possible for everyone to build a custom $3 \mathrm{D}$ printer for several hundred dollars. In 2000s a scientist at the Wake Forest Institute for Regenerative Medicine used a 3D printer to build a synthetic scaffold of a human bladder (1), which was coated with living cells and grew a functional organ, which was subsequently transplanted successfully into a living human host. This led to bioprinting - the technology to build a living tissue or organ.

\section{PRINCIPLES}

$3 \mathrm{D}$ printing is a technology, in which a computer-controlled robot (CNC machine) builds a threedimensional digital model by the deposition of the building material one layer after another, until the model is done. This process is called addictive manufacturing in contrast to subtractive manufacturing, in which the material is removed via machining techniques, such as milling, drilling, sawing etc. Both techniques can be used at the same time, with a single CNC (computer numerical control) machine. The machine operates in three axes $-\mathrm{X}$ and $\mathrm{Y}$ for the longitude and latitude of the active device and a $\mathrm{Z}$ axis for its depth. The axes can be arranged as a Cartesian coordinate system (XYZ, Cartesian robot) or they can be parallel to the active device (Delta robot). The CNC machine is driven by stepper motors or servo-motors, which provide highly accurate movements in the all axes. The whole mechanism of the $\mathrm{CNC}$ machine is enclosed in a large box as a safety measure and is $100 \%$ electronically controlled.

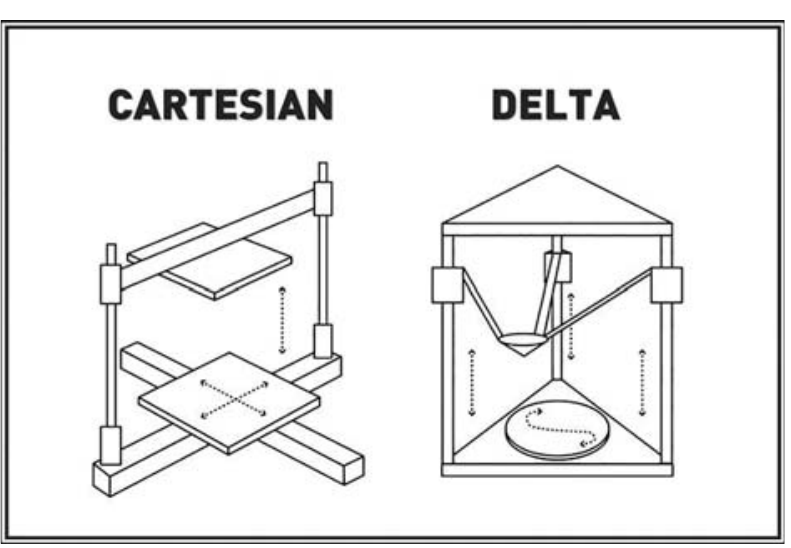

Fig. 1. Delta vs Cartesian

The CNC machine is highly automated. The movements of the three axes are defined by CAD software, and then translated into machine code by computer-aided manufacturing (CAM) software. The result is translated into the specific commands necessary for the building of the desired object and then loaded into the CNC machine. The complete list of operations, which can be performed by a CNC machine, includes 3D printing, laser cutting, welding, friction stir welding, ultrasonic welding, flame and plasma cutting, bending, spinning, hole-punching, pinning, gluing, fabric cutting, sewing, tape and fiber placement, routing, picking and placing, and sawing.

The 3D printer builds the objects layer by layer, from the bottom to the top. The height of the layers determines the resolution of the printer - thinner layers are used for objects with high resolution, but require longer building time, while thicker layers are used for objects with low resolution and the process is much faster. During the building of the object its layers and the $3 \mathrm{D}$ printer itself is subjected to the gravity force. To provide the necessary stability and to hold all the layers at their places, a special scaffold is included in the model and then it is removed during the completion of the object. The more scaffolds are included, the bigger the quality of the final product is. The scaffold can be built by water-solvent material, which can be easily removed with water after the print.

3D printing includes several similar technologies, which work with specific sets of materials:

$\diamond$ Selective laser sintering (SLS, SLA, DLP): A powerful laser is used to sinter powdered ma- 
terial (metal, photopolymer, ceramics, thermoplastics) at points in space defined by a CAD model, binding the material together layer by layer to create a solid structure. This method is quick and highly accurate - some SLS printers can achieve a layer height under 10 micrometers. The drawback is the limited variety of materials, which can be used in a single print.

$\diamond$ Extrusion (FDM, FFF, DIW): This is the most frequently used method of $3 \mathrm{D}$ printing. The material in the form of a gel is added by a syringelike device (extruder). The material can be extruded without a change of its initial form (hydrogels, creams, paste, clay) or it can be initially melted by the extrusion device and then extruded (thermoplastics, sugar, glass, chocolate etc.). After its deposition on the building plat- with ultrasound, piezoelectric solenoid or temperature. In this fashion the valve can print directly with cells (embryonic stem cells, induced pluripotent stem cells, somatic stem cells etc.), which can be added to an extracellular matrix, composed by natural or artificial polymers, prepared as a scaffold prior to printing or at the same time when extrusion is performed during the same print. Inkjetting keeps the cells alive and can position them precisely, resulting in artificial organ (organoid, tissue engineered construct, bioartificial organ). After the printing, the object can be placed in an incubator until the artificial extracellular matrix is replaces by the stem cells, the organ maturates and then it can be used in vitro for pharmaceutical and pathophysiological experiments or implanted in a living organism. a

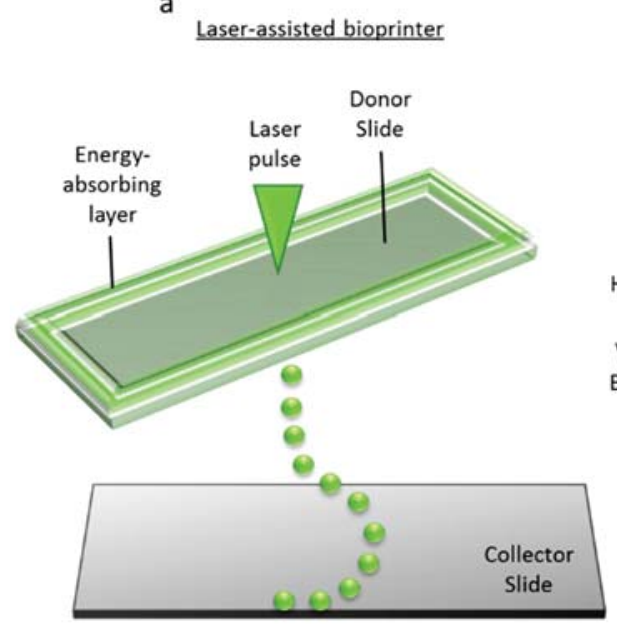

b

Inkjet bioprinter

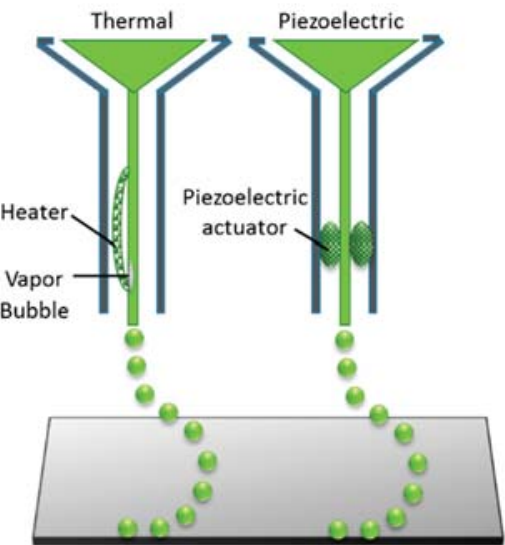

Fig. 2. Extrusion principle c Extrusion bioprinter

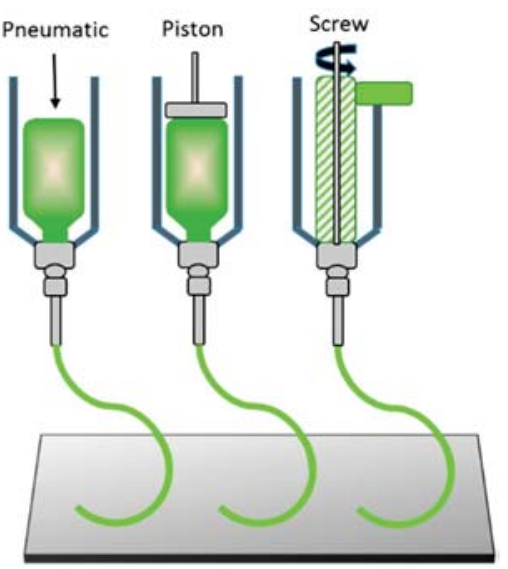

The initial design of the 3D printed objects

form, the melted material polymerizes into its final stage by cooling, chemical reaction, photocoagulation, etc. This method provides a vast range of materials - plastics, rubber, glass, silicone, clay, ceramics, metals, metal alloys and a wide range of composite (mixed) materials. A big advantage of this method is the extrusion of several materials (in some 3D printers up to 24) in a single print. The minimal layer height is between 100 and 10 micrometers.

$\diamond$ Inkjetting: This method is similar to the inkjet printers. The material is added as a solution, which is dispersed to droplets by a valve starts with a tomographic imaging (CT, MRI, PET, Z-stack, etc.), which can be translated into a 3D model by a medical computing software and then edited with a CAD software to an STL (stereolithography) model. Then the CAD model is translated to a CAM model and executed by the CNC machine. The robot can combine the additive manufacturing techniques with subtractive techniques as laser engraving, drilling, cutting, etc. until the model is done. Then the model has to be finished - the scaffolds have to be removed, the surface - polished, etc. The quality of the imaging study and the skill of the 3D program- 
mer, together with the technical characteristics of the CNC machine, determine the final outcome - anatomically correct, standardized 3D model, which can easily be 3D printed as a teaching model, medical device or tissue engineered organoid.

\section{APPLICATIONS}

The main purposes of $3 \mathrm{D}$ printed models in medicine are:

$\diamond$ Teaching model: The 3D printed model can be used for teaching or demonstration purposes by anatomists and clinicians alike.

$\diamond$ Training simulator: If the 3D model is printed with silicone or rubber, it can be used by the interventional specialists or the medical students as a training simulator for medical manipulations, surgical and interventional procedures. With more complex models a training operation can be performed with active circulation, beating heart and breathing lungs

$\diamond$ Pharmacological/pathophysiological tissue models: Theoretically, with $3 \mathrm{D}$ printing it is possible to create the extracellular matrix of virtually every tissue in the human body. Then the tissue model can be manufactured directly with stem cells or it can be inoculated additionally after the finalization of the print. Then the model can be incubated in special conditions until a certain stage of maturity and differentiation is achieved. Such 3D model can be used in pharmacological or pathophysiological studies instead of an experimental animal.

$\diamond$ Medical devices: The precision of the 3D printed models and the vast range of possible materials makes it an excellent candidate for personalized, custom medical tools and devices, made for the individual needs of the medical staff or for the anatomo-physiological characteristics of the particular patient.

$\diamond$ Bioartificial organs: The right combination of 3D printed extracellular matrix and stem cells results in a tissue-engineered organ, which can be implanted to a patient in order to replace a deceased, injured or missing one.

3D printing provides many opportunities for medical science, mainly because of its ability to create an accurate, living tissue or organ models. Such structures can be further augmented with electronics to provide additional properties, otherwise extraneous for the living organisms. At this stage, some simple organs are created as bioartificial grafts, including an external nose (2), auricula (3), skin (4), vascular grafts (5), ureter and bladder (1), trachea (6), etc. Advanced organs like kidneys, liver, hearth or brain cannot be created at this stage because of their anatomical and physiological complexity.

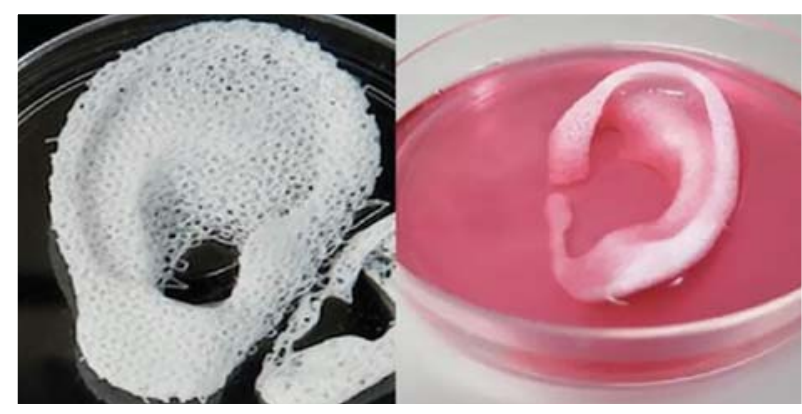

Fig. 3. Auricula

\section{CHALLENGES}

There are still some limitations, which slow the development of the bioartificial organs down:

$\diamond$ The manufacturing of complete and functional network of blood vessels in the bioartificial graft is still a challenge. The bioengineered tissues or organ models can develop necrotic core because of the inadequate supply of nutrients and oxygen, which makes only the creation of simple organs possible at this moment.

$\diamond$ The best layer height for the extrusion-based 3D printers is 10 micrometers, achieved only by some high-end machines, while most of the middle- and low-end 3D printers can achieve 50 micrometers at best. This makes the manufacturing of complex structures like kidney glomerulus or liver lobes impossible for now.

$\diamond$ A large multispecialist team of medical specialists, engineers and programmers is required.

$\diamond$ There are some ethical concerns, regarding the use of human stem cells, the implantations of such biomechanical devices into living hosts, and the possible complications of this process.

\section{CONCLUSION}

3D printing is a fast developing, cheap and promising technology with many applications in 
most industries, including medicine. For the year 2016 , the revenue of the 3D printing industry surpassed \$5.1 Billion worldwide and it keeps growing. Additive manufacturing is still in its early stages of developing, but shows great promise in the field of medicine as a method for the creation of realistic teaching or training models, pharmaceutical or pathophysiological experimental models, individualized medical tools and devices and bioartificial tissue or organ grafts for the need of implantology. There are still some issues regarding the lack of functional vascular network in the manufactured grafts, the limitations in the layer height of the created models, the requirement of a large multispecialist team and the bioethical concerns about the use of 3D printed devices in humans.

In the future, 3D printing will give us the ability to create functional organs, which can be further augmented with electronic or mechanical elements. Those organs will be better than our original ones and will give us abilities far beyond our wildest dreams. We will replace our original organs with synthetic ones willingly, making ourselves stronger, faster, smarter and longer living. Bioartificial heart, kidney, liver, brain... No one knows where this technology will lead and what will happen with humanity in the process, but one thing is certain - it will change medicine forever.

\section{Conflict of Interest}

The authors declare no conflict of interest

\section{REFERENCES}

1. Atala A, Bauer SB, Soker S, Yoo JJ, Retik AB. Tissue-engineered autologous bladders for patients needing cystoplasty. Lancet. 2006;367(9518):1241-6. doi: 10.1016/S0140-6736(06)68438-9

2. Xu Y, Fan F, Kang N, Wang S, You J, Wang H, et al. Tissue engineering of human nasal alar cartilage precisely by using three-dimensional printing. Plast Reconstr Surg. 2015;135(2):451-8. doi: 10.1097/ PRS.0000000000000856

3. Nuseir A, Hatamleh M, Watson J, Al-Wahadni AM, Alzoubi F, Murad M. Improved Construction of Auricular Prosthesis by Digital Technologies. J Craniofac Surg. 2015;26(6):e502-5. doi: 10.1097/ SCS.0000000000002012

4. Min D, Lee W, Bae IH, Lee TR, Croce P, Yoo SS. Bioprinting of biomimetic skin containing mela- nocytes. Exp Dermatol. 2017 ;00:1-7. doi: 10.1111/ exd.13376

5. Pinnock CB, Meier EM, Joshi NN, Wu B, Lam MT. Customizable engineered blood vessels using 3D printed inserts. Methods. 2016;99:20-7. doi: 10.1016/j.ymeth.2015.12.015

6. Chang JW, Park SA, Park JK, Choi JW, Kim YS, Shin YS, et al. Tissue-engineered tracheal reconstruction using three-dimensionally printed artificial tracheal graft: preliminary report. Artif Organs. 2014;38(6):E95-E105. doi: 10.1111/aor.12310 